\title{
Do you know how to get the J-START quality assurance guideline?
}

\author{
Takayoshi Uematsu $^{1,3} \cdot$ Seigo Nakamura ${ }^{1,4} \cdot$ Noriaki Ohuchi $^{2,5}$
}

Received: 23 March 2018 / Accepted: 24 April 2018 / Published online: 9 May 2018

(c) The Japanese Breast Cancer Society 2018

The Japan Strategic Anti-cancer Randomized Trial (J-START), which is the world's first large-scale randomized controlled trial of supplemental screening breast ultrasonography in asymptomatic women aged 40-49 years, which has being conducted in Japan, assessed the efficacy of mammography with adjunctive ultrasonography in improving sensitivity and detection rates of early breast cancers in The Lancet [1]. Preliminary results from the J-START showed that the sensitivity was significantly higher in the intervention group than in the control group (91.1 vs. $77.0 \% ; p=0.0004)$, whereas the specificity was significantly lower ( 87.7 vs. 91.4\%; $p<0.0001)$. More cancers were detected in the intervention group than in the control group [184 $(0.50 \%)$ vs. 117 $(0.32 \%) ; p=0.0003]$ and were more frequently stages 0 and 1 [144 (71.3\%) vs. 79 (52.0\%); $p=0.0194]$. Furthermore, there was a significant reduction in interval cancers $(0.05$ vs. $0.10 \% ; p=0.034)$. Although the mortality rates are the most important parameters to evaluate the efficacy of supplemental screening breast ultrasonography, preliminary results from the J-START are essential in informing personalized supplemental screening modality choices for women with dense breasts.

Breast ultrasonography is highly operator-dependent, requiring real-time adjustments of gain, dynamic range, contrast, depth of field, examiner and patient positioning, recognition of abnormalities, etc. Therefore, the quality control is very important, especially, in population-based breast

Takayoshi Uematsu

t.uematsu@scchr.jp

Japanese Breast Cancer Society, Tokyo, Japan

2 Japan Association of Breast Cancer Screening, Tokyo, Japan

3 Department of Breast Imaging and Breast Intervention Radiology, Shizuoka Cancer Center Hospital, Shizuoka, Japan

4 Division of Breast Surgical Oncology, Department of Surgery, Showa University Hospital, Tokyo, Japan

5 Tohoku University Graduate School of Medicine, Miyagi, Japan cancer screening programs. The J-START was initiated to not only verify effectiveness of adjunctive ultrasonography, but also standardize ultrasonography equipment, examination method, and the interpretation criteria in breast cancer screening [2]. In The Lancet [1], the authors did not describe their quality assurance guideline applied in the J-START because of word limitation reasons. They have kept it in mind that good quality control of breast ultrasonography systems and methods is critical to keep screening associated harms to a minimum. Therefore, they prepared the J-START quality assurance guideline including quality control for adjunctive ultrasonography systems, educational and training programs, and interpretation criteria before start of the J-START trial. Except for the J-START quality assurance guideline, we did not identify any appropriate, completed adjunctive ultrasonography quality assurance guideline in terms of quality assurance of adjunctive ultrasonography for breast cancer screening. The quality assurance guideline for adjunctive ultrasonography in breast cancer screening [3] has eight chapters, going through standards for equipment, examination methods, image reading and evaluation, educational and training programs, and ending up with a chapter on qualifications of referrals. This guideline will be most useful as a reference guide for supplemental screening breast ultrasonography, and some of the chapter, especially educational and training programs section, is worth reading for gaining a basic understanding of the introduction of adjunctive ultrasonography in breast cancer screening. The quality assurance guideline for adjunctive ultrasonography in breast cancer screening is a resource worth reading. Please feel free to download it at http://jbcs.gr.jp/men/english/ on Japanese Breast Cancer Society website. The J-START was support by the third Comprehensive Control Research for Cancer programme of the Japanese Ministry of Health, Labour and Welfare.

\section{Compliance with ethical standards}

Conflict of interest Takayoshi Uematsu, Seigo Nakamura, and Noriaki Ohuchi have no COI. 


\section{References}

1. Ohuchi N, Suzuki A, Sobue T, Kawai M, Yamamoto S, Zheng YF, et al. Sensitivity and specificity of mammography and adjunctive ultrasonography to screen for breast cancer in the Japan Strategic Anti-cancer Randomized Trial (J-START): a randomised controlled trial. Lancet. 2016;387:341-8.

2. Ishida T, Suzuki A, Kawai M, Narikawa Y, Saito H, Yamamoto $\mathrm{S}$, et al. A randomized controlled trial to verify the efficacy of the use of ultrasonography in breast cancer screening aged 40-49 (J-START): 76196 women registered. Jpn J Clin Oncol. 2014;44:134-40.

3. Tohno E, Ohuchi N, Obane N, Sakurai M, Shirai H, Tsunoda $\mathrm{H}$, et al. Quality assurance guideline for adjunctive ultrasonography in breast cancer screening. Grant in aid for innovative cancer research funded by Japan Agency for Medical Research and Development (AMED), Tokyo, 2016. http://jbcs.gr.jp/men/engli sh/. Accessed 4 May 2018. 